\title{
Genome-wide deleterious mutation favors dispersal and species integrity
}

\author{
MT Morgan \\ School of Biological Sciences, Washington State University, WA, USA
}

Here I develop the idea that ubiquitous harmful genomewide mutation with local differentiation favors dispersal, even though migration reduces average fitness. Historical contingency of the mutational process means that demes (subpopulations) differentiate from one another. Deleterious or lethal partially recessive mutations carried by migrants then do not encounter similar mutations in the recipient deme.
Migrant offspring have higher fitness than offspring of residents, because migrant offspring are heterozygous rather than homozygous for harmful mutations. The advantage is inversely related to local inbreeding depression. Genomewide deleterious mutation favors the evolution of dispersal, which in turn enhances the genetic integrity of the species. Heredity (2002) 89, 253-257. doi:10.1038/sj.hdy.6800143

Keywords: deleterious mutation; dispersal; migration; inbreeding; theory

\section{Introduction}

There are many environmental (eg, Van Valen, 1971; Comins et al, 1980; Levin et al, 1984) and genetic (eg, Hamilton and May, 1977; Shields, 1987) explanations for why organisms disperse. Shields (1987) argues, in part, that dispersal is a mechanism for avoiding reduced fitness due to inbreeding depression; this mechanism is illustrated by the recent study by Ebert et al (2002; see also Ives and Whitlock, 2002). Inbreeding depression in natural populations of plants (Husband and Schemske, 1996) and animals (Crnokrak and Roff, 1999) is due in large degree to harmful mutations (Charlesworth and Charlesworth, 1999). Two broad classes of harmful mutations affect fitness and inbreeding depression. Deleterious mutations are weakly selected and partly recessive (Garcia-Dorado and Caballero, 2000; eg, heterozygous fitness $1-h s$, homozygous fitness $1-s$, dominance coefficient $h=0.1$, selection coefficient $s=0.1$ ). Lethal mutations are strongly selected and very recessive (eg, $h=0.02, s=1)$. Estimates of the harmful mutation rate per genome per generation are highly controversial (Lynch et al, 1999), but in experimental populations range between $U=0.01$ and 1 for deleterious mutations, and between $U=0.001$ and 0.1 for lethal mutations (Drake et al, 1998). Despite the relatively sound understanding of the genetic basis of inbreeding depression, explicit genetic models for the evolution of dispersal in the face of inbreeding depression have not been developed.

The primary goal of this paper is to show that genomewide deleterious mutation provides a substantial advantage to migration. Analytic calculations are used to explore the effects of mutation and selection parameters, particularly in relation to consequences for migrant rela-

Correspondence: MT Morgan, School of Biological Sciences, Washington State University, Pullman, WA 99164-4236, USA.

E-mail:mmorgan@wsu.edu

Received 5 December 2001; accepted 18 June 2002 tive fitness and local inbreeding depression. Calculations are supported, and extended to include the effects of recombination, through explicit simuations. Finally, modifiers are incorporated into the simulation, to confirm that individual selection favors migration under genomewide deleterious mutation.

\section{Methods}

\section{Mathematical analysis}

Wright's diffusion approach and the assumption of multiplicative fitness interactions between loci can be used to develop expressions for mean fitness of crosses within and between demes in an island model (Whitlock et al, 2000). The diffusion leads to a distribution of allele frequencies $p$ within demes equal to

$$
\Psi(p)=C p^{4 N m p-1}(1-p)^{4 N \mu+4 N m(1-\bar{p})-1} \bar{w}^{2 N}
$$

where $N$ is deme size, $\mu$ the deleterious mutation rate per locus, $m$ the migration rate between demes, and $\bar{p}=E(p)$ the average allele frequency over all demes. The constant $C$ is chosen so that $\int_{0}^{1} \Psi(p) \mathrm{d} p=1$. It is not possible to solve this distribution explicitly, but solution via numerical integration is easily accomplished (Kimura et al, 1963) using Mathematica (Wolfram Research Inc, 2000; notebook available on request). The distribution of allele frequencies between demes can be used to calculate expected values $\mathrm{E}[p], \mathrm{E}\left[p^{2}\right]$. At a single locus, the expected mean fitness of migrant, local (ie, non-migrant), and inbred (self-fertilized) progeny are:

$$
\begin{aligned}
\bar{w}_{\text {migrant }} & =1-2 \mathrm{E}[p](1-\mathrm{E}[p]) h s-(1-\mathrm{E}[p])^{2} s \\
\bar{w}_{\text {local }} & \left.=1-2 \mathrm{E}[p(1-p)] h s-\mathrm{E}(1-p)^{2}\right] s \\
\bar{w}_{\text {inbred }} & =1-\mathrm{E}[p(1-p)] h s-\mathrm{E}[(1-p)(2-p)] s / 2
\end{aligned}
$$

Assuming multiplicative fitnesses across $n$ loci with identical mutational and fitness properties results in fitnesses with the form

$$
\bar{W}_{\text {migrant }} \approx(1-h s)^{2 \mathrm{E}[p](1-[p]) n}(1-s)^{(1-\mathrm{E}[p])^{2} n}
$$




$$
\begin{aligned}
\bar{W}_{\text {local }} & \approx(1-h s)^{2 \mathrm{E}[p](1-\mathrm{E}[p]) n}(1-s)^{(1-\mathrm{E}[p])^{2} n} \\
\bar{W}_{\text {inbred }} & \approx(1-h s)^{\mathrm{E}[p(1-p)] n}(1-s)^{\mathrm{E}(1-p)(2-p) n / 2}
\end{aligned}
$$

(cf, Whitlock et al, 2000). I define migrant relative fitness as $\bar{W}_{\text {migrant }} / \bar{W}_{\text {local }}$, and local inbreeding depression as $1-\bar{W}_{\text {inbred }} / \bar{W}_{\text {local }}$. The animal dispersal literature frequently defines 'inbreeding depression' as the reduction in fitness of local compared to migrant matings (see references in Clobert et al, 2001); this measure is more similar (ie, proportional) to migrant relative fitness, rather than to local inbreeding depression as used here.

Insight into migrant relative fitness comes from rearranging Whitlock's equations. The advantage to migration in the face of deleterious mutation to partially recessive deleterious alleles at $n$ identical loci is

$$
\frac{\bar{W}_{\text {migrant }}}{\bar{W}_{\text {local }}}=\left(\frac{(1-h s)^{2}}{1-s}\right)^{n V(p)}
$$

$V(p)$ is the equilibrium between-deme variance in allele frequency. The migrant relative fitness from fully recessive lethal mutations is $\bar{W}_{\text {migrant }} / \bar{W}_{\text {local }}=e^{n V(p)}$. Migrant relative fitness increases with the between-population variation in allele frequency, $V(p)$. Increasing the number of loci experiencing deleterious mutation, and hence the genome-wide deleterious mutation rate, enhances migrant relative fitness.

\section{Simulation of equilibrium populations}

Analyses involve discrete-generation individual-based simulations (Charlesworth et al, 1992). Simulated populations consist of $D$ demes, each with $N$ monoecious individuals. Each individual contains two 'gametes' (strings of 0 's and 1's; 1's correspond to deleterious alleles) with $n=1024$ loci. Offspring result from 'local' or 'migrant' mating, depending on the propensity of parental individuals to produce migrant offspring. The probability of offspring migration is $m$ in simulations without migration rate modifiers; it is a function of deme and genotype frequencies in modifier simulations. Local offspring are formed by mating parents within the deme. Choice of maternal parent is proportional to $1-m$ in equilibrium simulations; it is deme and genotype specific in modifier simulations. Choice of paternal parent is uniformly distributed over all individuals in the maternal deme. Migrant offspring are formed by mating a maternal parent selected from the entire population. Choice of random maternal parent is proportional to $m$ in equilibrium populations; it is genotype-specific in modifier simulations. Choice of paternal parent is uniformly distributed over all individuals in the maternal deme. The simulation is thus constructed so that, in the absence of deleterious mutation, genotypes with higher migration have no intrinsic advantage.

Mating involves gamete and offspring formation, and selection based on offspring fitness. Gametes are created by generating a recombination map, and using this map to select alleles at each locus. The map has a Poissondistributed number $(\bar{R}=2)$ of randomly placed recombination events. Each gamete experiences a Poisson-distributed number (mean $=U / 2$ ) of randomly placed mutations to deleterious alleles. Gametes generated from each of the parents form the offspring genotype. Offspring with $i$ heterozygous and $j$ homozygous alleles have fitness $w_{i j}=(1-h s)^{i}(1-s)^{j}$. Offspring are incorpor- ated into the next generation when $w_{i j}$ exceeds a uniform $[0,1)$ random number.

Equilibrium populations are characterized by timeaveraging. A population runs for 2000 generations. The population is then characterized every 100 generations, until generation 12000. Each characterization involves 10 within- and 10 between-deme offspring generated for each individual. Results are the average over all individuals and generations.

\section{Modifiers of the migration rate}

Modifier simulations involve repeated introduction of modifier alleles into quasi-equilibrium (Kondrashov, 1995) populations. The population state after 2000 generations is recorded. A single modifier, with migration rate $m^{\prime}$, is then introduced and followed until its frequency becomes 0 or exceeds $p_{\text {crit }}=0.025$. The population state prior to modifier introduction is then restored, and another modifier introduced. This is repeated 100 independent times. The population state prior to modifer introduction is then restored once again, iterated for 200 generations, the new population state recorded, and a second series of 100 modifiers introduced. This is repeated 1000 times, for 100000 total introductions for each set of parameter values. The frequency of loss and passage above $p_{\text {crit }}$ are compared to neutral modifiers (ie, modifiers with migration rates identical to the resident type). An approximate measure of selection is the probability of first passage of 'selected' modifiers divided by the probability of first passage of neutral modifiers, $s_{m^{\prime}}=\left(1-P_{\text {loss } \mid m^{\prime}}\right) /\left(1-P_{\operatorname{loss} \mid m^{\prime}=\mathrm{m}}\right)-1$

\section{Results}

Genome-wide mutation-selection balance calculations in structured populations are illustrated in Figure 1. Reduced migration decreases the average number of deleterious alleles in the population. This is because restricted migration increases local inbreeding, generating homozygotes that are easily targeted by selection. The decrease in number of deleterious alleles is accompanied by a very modest increase in population mean fitness, and more pronounced decrease in local inbreeding depression. The lower local inbreeding depression might be surprising, since decreasing migration increases relatedness within demes. As anticipated by equation 2, the fitness of offspring of migrant individuals increasingly exceeds the fitness of offspring of local (ie, nonmigrant) individuals as migration rate decreases and between deme variance in allele frequency increases. Migrant individuals carry different deleterious alleles than local individuals, the more so as population differentiation increases (Crow, 1948). Offspring of migrants therefore benefit from heterozygosity, rather than homozygosity, for deleterious alleles. Similar results are observed for recessive lethals, although lower lethal mutation rates reduce the magnitude of the advantage (Table 1). Results suggest a substantial advantage to increasing migration.

Deme size influences equilibrium allele number, fitness, and the advantage to migration (Figure 2). The average number of deleterious alleles decreases with deme size, provided deme size is moderate (eg, $N>50$ ). This is because inbreeding and consequent homozygosity in small demes allows expression, and selective removal, of 


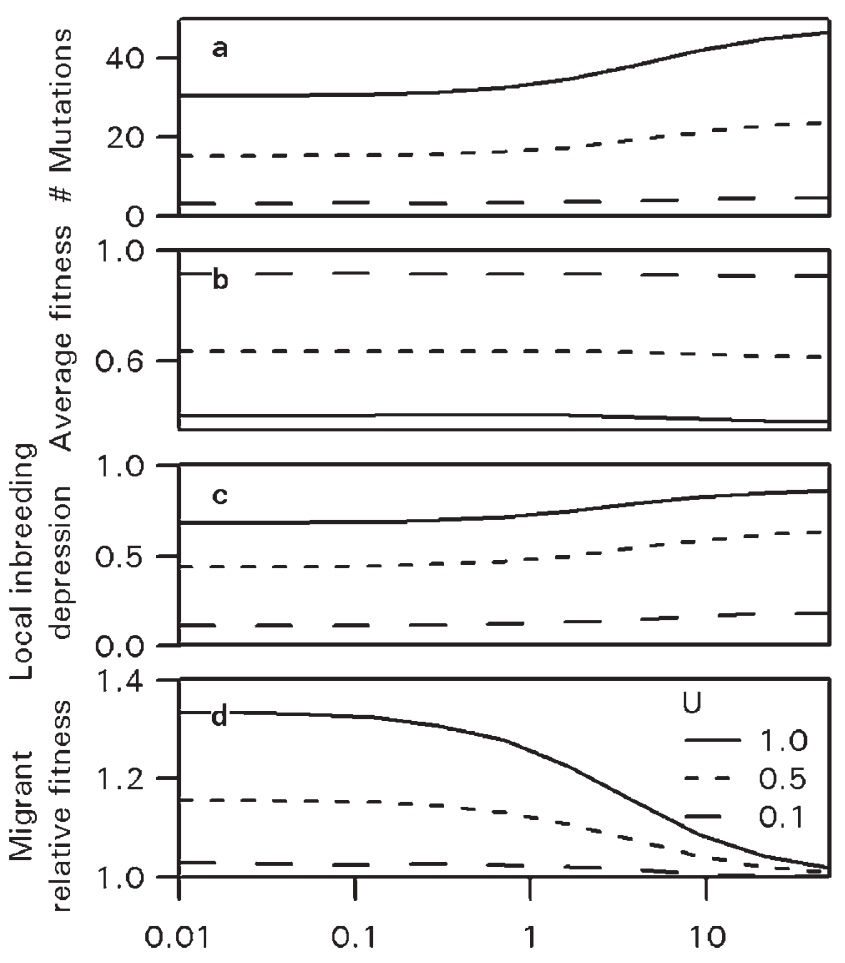

Number of migrants per deme per generation

Figure 1 Population-level advantage to migrants under various levels of mutation and migration. Calculations assume an infinite number of demes with $N=1000$ each. Fitness is determined by genomic mutation at rate $U$ to deleterious $(h=0.1, s=0.1)$ alleles at $n=10,000$ loci. The panels show total number of mutations per gamete, $\bar{n}$, population average fitness, $\bar{W}$, local inbreeding depression ( $\left.1-\bar{W}_{\text {inbred }} / \bar{W}_{\text {local }}\right)$, and average fitness of offspring of migrant compared to local parents $\left(\bar{W}_{\text {migrant }} / \bar{W}_{\text {local }}\right)$.

Table 1 Influence of parameter values on number of deleterious alleles $\bar{n}$, and fitness $\bar{W}$ of local and migrant individuals. Calculations assume demes of $N=100$ individuals and migration at rate $N m=1$. Lethal recessive parameters are $U=0.1, h=0, s=1$, with $n=10000$ loci. Standard parameters are $U=1, h=0.1, s=0.1$, with $n=1024$ loci. Reduced number of loci in the standard parameters facilitate comparison with computationally intensive simulations

\begin{tabular}{llrccc}
\hline Parameter & Value & $\bar{n}$ & $\bar{W}_{\text {local }}$ & $\bar{W}_{\text {migrant }}$ & $\bar{W}_{\text {migrant }} / \bar{W}_{\text {local }}$ \\
\hline Lethal recessive & & 1.43 & 0.948 & $\approx 1$ & 1.05 \\
Standard & & 30.3 & 0.416 & 0.503 & 1.21 \\
Dominance, $h$ & 0.05 & 37.0 & 0.461 & 0.607 & 1.32 \\
& 0.2 & 21.4 & 0.373 & 0.408 & 1.09 \\
Selection, $s$ & 0.3 & 16.1 & 0.355 & 0.370 & 1.04 \\
& 0.01 & 187.8 & 0.426 & 0.520 & 1.22 \\
& 0.025 & 92.3 & 0.425 & 0.532 & 1.25 \\
Locus number, $n$ & 0.05 & 53.0 & 0.423 & 0.525 & 1.24 \\
& 0.2 & 17.2 & 0.402 & 0.473 & 1.18 \\
Recombinations & 512 & 27.9 & 0.426 & 0.501 & 1.17 \\
per gamete, $\bar{R}$ & 4096 & 32.7 & 0.406 & 0.507 & 1.25 \\
& 0.5 & 33.2 & 0.403 & 0.508 & 1.26 \\
& 1 & 39.2 & 0.324 & 0.376 & 1.16 \\
& 2 & 34.3 & 0.376 & 0.435 & 1.16 \\
& 4 & 31.8 & 0.380 & 0.443 & 1.17 \\
& Free & 31.4 & 0.403 & 0.468 & 1.16 \\
& & & & 0.474 & 1.17 \\
\hline
\end{tabular}

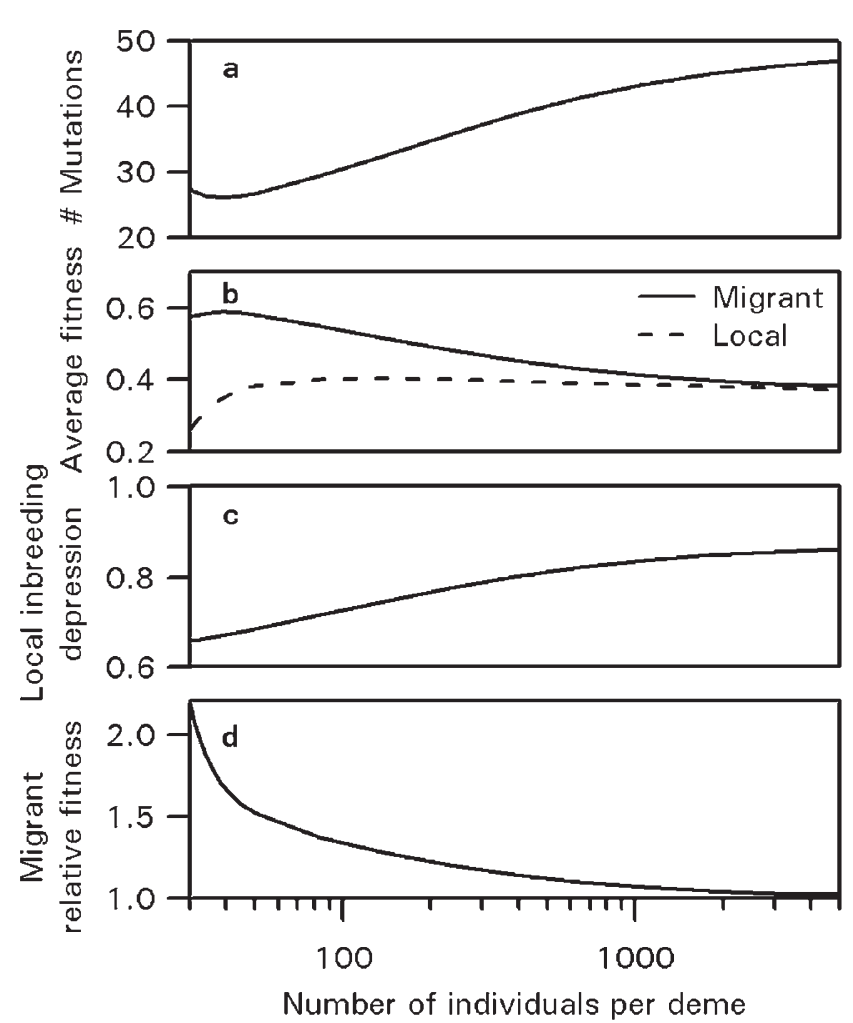

Figure 2 Consequences of deme size for migrant fitness advantage, with migration $m=0.01$. Fitness is determined by genomic mutation $(U=1.0)$ to deleterious $(h=0.1, s=0.1)$ alleles at $n=10,000$ loci. The panels show total number of mutations per gamete, $\bar{n}$, population average fitness, $\bar{W}$, local inbreeding depression $\left(1-\bar{W}_{\text {inbred }} / \bar{W}_{\text {local }}\right)$, and average fitness of offspring of migrant compared to local parents $\left(\bar{W}_{\text {migrant }} / \bar{W}_{\text {local }}\right)$.

recessive alleles. Despite these purifying effects of inbreeding, fitness of local individuals (and population average fitness) decreases with deme size. This is because selection becomes less effective as population size decreases. On balance, the increased exposure of recessive alleles caused by inbreeding does not compensate for the reduced power of selection due to finite population size. The average fitness of migrant individuals increases with decreasing deme size. In large demes, two local individuals are as likely to share different deleterious alleles as are migrant and local individuals. In smaller demes, drift increases differentiation between demes so that local individuals share deleterious alleles more than do migrant individuals. Migrant fitness eventually decreases as demes become very small (Figure 2). The fitness advantage of migrants increases with decreasing deme size, becoming dramatic in small demes (eg, $N<50$ ).

Effects of dominance, selection, and locus number are summarized in Table 1 (also Whitlock et al, 2000; Whitlock, 2002). Greater dominance increases the equilibrium number of deleterious alleles, local and migrant fitness, and the advantage to migrants. Deleterious alleles increase in frequency because dominance shelters them from selection. Paradoxically, local mean fitness increases with dominance in structured populations because deleterious alleles are efficiently eliminated in pairs (ie, as homozygotes) (Haldane, 1957; Kimura and Maruyama, 1966). Weaker selection increases the equilibrium number of deleterious alleles and local mean fitness. Weaker 
selection initially increases migrant fitness and advantage, but as selection becomes very weak migrant fitness and advantage start to decrease. The large number of weakly selected mutations results in decreased allele frequency variance between populations, and hence reduced migrant relative fitness. Decreasing locus number decreases the number of deleterious alleles, local and migrant fitness, and the migrant relative fitness. The effects on number of alleles and local fitness are counterintuitive, since fewer loci imply stronger mutation at each locus. Usually substantial migrant advantage occurs for all parameter values in Table 1 .

Simulations support the general conclusions of the analysis while illustrating the effects of recombination (Table 1). Migrant relative fitness in simulations parallels, at slightly reduced levels, the relative fitness seen in numerical calculations. The reduction in migrant relative fitness occurs because the finite number of simulated demes increase the probability of identity by descent between deleterious alleles in migrant mating. Introducing restrictions on recombination $R$ decreases fitness, because less recombination increases selective interference between loci (Hill and Robertson, 1966). Rates of recombination below $R \approx 0.5$ per gamete (results not shown) dramatically increase numbers of alleles and decrease local and migrant mean fitness; low recombination facilitates a ratchet-like increase in allele number (Charlesworth et al, 1992; Higgins and Lynch, 2001). A similar ratchet-like effect occurs in very small demes (results not shown).

Simulations verify that the advantage to migration illustrated in Figure 1 translates into selection on modifiers of the migration rate (Table 2). Dominant single locus modifiers decreasing the migration rate are more likely, and modifiers increasing the migration rate less likely, to be lost than neutral modifiers. Selection on modifiers is subtle, involving hitch-hiking in high-fitness heterozygous progeny in early generations after a migration event. Modifier fitness advantage thus depends on linkage with harmful mutations; the most pesimistic situation (an unlinked modifier) is assumed here. For fixed modifier effect $\left(\mathrm{eg}, m^{\prime}=m \pm 0.05\right)$, the

Table 2 Selection of modifiers of the migration rate. Dominant alleles modifying the migration rare are introduced 100,000 times into quasi-equilibrium populations of $N=100$ individuals in each of $D=10$ demes. The genome-wide deleterious mutation rate to deleterious, partially recessive $(h=0.1, s=0.1)$ mutations is $U=1.0$. The table reports probability of loss, $P_{\text {loss }}$ and apparent selection on the modifiers, $s_{m^{\prime}}$.

\begin{tabular}{lccc}
\hline Resident, $m$ & Modifier, $m^{\prime}$ & $P_{\text {loss }}$ & $s_{m^{\prime}}$ \\
\hline 0.1 & $m-0.05$ & 0.9835 & -0.123 \\
& $m$ & 0.9812 & \\
0.2 & $m+0.05$ & 0.9799 & 0.067 \\
& $m-0.05$ & 0.9820 & -0.050 \\
0.3 & $m$ & 0.9811 & \\
& $m+0.05$ & 0.9794 & 0.088 \\
& $m-0.05$ & 0.9818 & -0.011 \\
0.4 & $m$ & 0.9816 & \\
& $m+0.05$ & 0.9812 & 0.017 \\
& $m-0.05$ & 0.9819 & -0.035 \\
& $m$ & 0.9813 & \\
& $m+0.05$ & 0.9805 & 0.042 \\
\hline
\end{tabular}

advantage to migration is expected to increase with migration rate. This is because selection acts on the product of migrant relative fitness (Figure 1) and fraction of migrant offspring; this number increases with migration rate. Simulations did not detect this subtle outcome.

\section{Discussion}

Inbreeding avoidance is a component of Shields' (1987) argument for optimal outbreeding. Shields does not explicitly consider genome-wide deleterious mutation as the basis of inbreeding depression. Emphasizing inbreeding avoidance as an explanation for migrant advantage may be misleading, for several reasons. Migrant relative fitness is greatest when local inbreeding depression is smallest (Figure 1). Greater inbreeding through restricted migration (Figure 1) and decreased deme size (Figure 2) decreases equilibrium allele number and increases population mean fitness, yet enhances migrant relative fitness. Migrant fitness usually exceeds panmictic expectation, showing the advantage to migration per se. Finally, simulation results emphasize the role of genetic drift through selective interference in small populations or with tight linkage.

The evolution of dispersal is partly analogous to the evolution of sex and outcrossing, in that migration is a mechanism for structuring genetic variation within and between individuals. Kondrashov (1993) provides a fourpart classification of hypotheses for the evolution of sex: mutational deterministic, mutational stochastic, environmental deterministic, and environmental stochastic. Many previous explanations for dispersal focus on environmental stochastic explanations, where migration is 'clearly advantageous' (Hamilton and May, 1977). The genome-wide deleterious mutational hypothesis is deterministic; a hint of mutational stochastic importance is seen in fitness decline in extremely small demes under restricted rates of genomic recombination (Higgins and Lynch, 2001). The particular appeal of mutational deterministic explanations is their universal application, provided genome-wide mutation is large. Unlike the evolution of sex or self fertilization, migration appears always to offer an advantage; the effects of purging are not sufficient to promote restricted dispersal (Figures 1 and 2; Table 2). Ironically, increasing migration undermines the fitness advantage of sexual compared to asexual reproduction (Agrawal and Chasnov, 2001).

Population mean fitness sometimes provides guidance for selection of modifiers (Karlin and McGregor, 1974). Results presented here show that reduced migration usually decreases the frequency of recessive alleles and increases mean fitness (Whitlock et al, 2000). This might suggest an advantage to reduced migration, analogous to the advantage to self-fertilization resulting from 'purging' of the mutational load (Lande and Schemske, 1985; Charlesworth et al, 1990). However, migrants experience an immediate fitness advantage (Figure 1) that translates into selection for increased migration (Table 2). Population mean fitness does not provide an adequate guide to migrant modifier selection.

High short-term fitness of migrant individuals might suggest conservation management programs involving between-population crosses or transplants, but this might be ill-advised. Successful migrants introduce many new deleterious alleles. Eliminating these alleles imposes a 
substantial genetic load on the deme that, in the long term, counterbalances the initial fitness advantage. Because the migrant alleles are more likely than resident alleles to be eliminated as heterozygotes than as homozygotes, migrant individuals are likely to make a small negative contribution to population-level fitness. Indeed, genome-wide mutation is of practical importance because, unlike stochastic events in natural populations, it is not possible to separately manage organisms and their genes. Finally, gene flow easily swamps local adaptation and prevents population divergence (Felsenstein, 1979; Coyne et al, 1997). This provides an explanation for why species maintain their genetic integrity during anagenic change, but does not explain the prevalence of migration. Harmful mutations provides a universal explanation for migration and hence the ultimate integrity of species.

\section{Acknowledgements}

Alexey Kondrashov and Michael Whitlock commented on earlier versions of this manuscript; the US National Science Foundation (DEB 9974017) provided financial support.

\section{References}

Agrawal AF, Chasnow JR (2001). Recessive mutations and the maintenance of sex in structured populations. Genetics 148: 912-665.

Charlesworth B, Charlesworth D (1999). The genetic basis of inbreeding depression. Genet Res 74: 329-340.

Charlesworth D, Morgan MT, Charlesworth B (1990). In-breeding depression, genetic load and the evolution of outcrossing rates in a multi-locus system with no linkage. Evolution 44: 1469-1489.

Charlesworth D, Morgan MT, Charlesworth B (1992). The effect of linkage and population size on inbreeding depression due to mutational load. Genet Res 59: 49-61.

Clobert J, Danchin E, Dhondt AA, Nichols JD (2001). Dispersal. Oxford University Press: Oxford.

Comins HN, Hamilton WD, May RM (1980). Evolutionary stable dispersal strategies. J theor Biol 28: 205-230.

Coyne JA, Barton NH, Turelli M (1997). Perspective: a critique of Sewall Wright's shifting balance theory of evolution. Evolution 51: 643-671.

Crnokrak P, Roff DA (1999). Inbreeding depression in the wild. Heredity 83: 260-270.

Crow JF (1948). Alternative hypotheses of hybrid vigor. Genetics 33: 477-487.

Drake JW, Charlesworth B, Charlesworth D, Crow JF (1988).

Rates of spontaneous mutation. Genetics 3148: 1667-1686.

Ebert D, Haag C, Kirkpatrick M, Riek M, Hottinger JW, Pajunen VI (2002). A selective advantage to immigrant genes in a Daphnia metapopulation. Science 295: 485-488.

Felsenstein J (1979). Excursions along the interface between disruptive and stabilizing selection. Genetics 93: 773-795.

Garcia-Dorado A, Caballero A (2000). On the average coefficient of dominance of deleterious spontaneous mutations. Genetics 155: 1991-2001.

Haldane JBS (1957). The cost of natural selection. J Genet 55: 511-524.

Hamilton WD, May RM (1977). Dispersal in stable habitats. Nature 269: 578-581.

Higgins K, Lynch M (2001). Metapopulation extinction caused by mutation accumulation. Proc Nat Acad Sci USA 98: 29282933.

Hill WD, Robertson A (1966). The effect of linkage on the limit to artificial selection. Genet Res 8: 269-294.

Husband BC, Schemske DW (1996). Evolution of the magnitude and timingof inbreeding depression in plants. Evolution 50: $54-70$.

Ives AR, Whitlock MC (2002). Inbreeding and metapopulations. Science 295: 454-488.

Karlin S, McGregor J (1974). Towards a theory of the evolution of modifier genes. Theor Popul Biol 5: 59-103.

Kimura M, Maruyama T (1966). The mutational load with epistatic gene interactions in fitness. Genetics 54: 1337-1351.

Kimura M, Maruyama T, Crow JF (1963). The mutation load in small populations. Genetics 48: 1303-1312.

Kondrashow AS (1993). Classification of hypotheses on the advantage of amphimixis. J Hered 84: 372-387.

Kondrashow A (1995). Modifiers of mutation-selection balance general approach and the evolution of mutation rates. Genet Res 66: 53-69.

Lande R, Schemske DW (1985). The evolution of self-fertilization and inbreeding depression in plants. I. Genetic models. Evolution 39: 24-40.

Levin DA, Cohen D, Hastings A (1984). Dispersal strategies in patchy environments. Theor Popul Biol 26: 165-191.

Lynch M, Blanchard J, Houle D, Kobota TT, Schultz ST, Vassilieva L, Willis JH (1999). Perspective: spontaneous deleterious mutation. Evolution 55: 645-663.

Shields WM (1987). Optimal inbreeding and evolution of philopatry. In: Swingland IR, Greenwood PJ (eds) The Ecology of Animal Movement, Clarendon Press. pp. 132-159.

Van Valen L (1971). Group selection and the evolution of dispersal. Evolution 25: 591-598.

Whitlock MC (2002). Selection, load and inbreeding depression in a large metapopulation. Genetics 160: 1191-1202.

Whitlock MC, Ingvarsson PK, Hatfield T (2002). Local drift load and the heterosis of interconnected populations. Heredity 84 : 452-457.

Wolfram Research 2000. Mathematica Version 4.1. Champaign: Illinois. 